\title{
Acute Ischemic Stroke Following Chemotherapy for Malignant Mixed Testicular Germ Cell Tumour: Does Cisplatin Play a Role?
}

\author{
(1) Berk Hazır, (1) Meylis Artykov, (1) Ahmet Aşcı, (1) Hakan Bahadır Haberal, (1) Mustafa Sertaç Yazıcı \\ Hacettepe University Faculty of Medicine, Department of Urology, Ankara, Turkey
}

\begin{abstract}
Among males aged between 15 and 35 years, testicular cancer is the most commonly diagnosed cancer. Testicular germ cell tumours are generally considered curable and respond dramatically to adjuvant treatment. Cisplatin-based chemotherapy regimens are used ubiquitously, inevitably leading to iatrogenic morbidity. Herein, we represent a case of a patient diagnosed with a non-seminomatous germ cell tumour, who underwent adjuvant cisplatin-based chemotherapy treatment and subsequently developed an acute ischemic stroke. In cancer patients, a malignancy-induced hypercoagulability state can cause thromboembolic events. Nonetheless, anti-cancer therapy may dramatically increase the risk of thromboembolic events, by analogue mechanisms, such as the release of pro-coagulant mediators, direct endothelial injury or stimulation of tissue factor production by host cells. Among various chemotherapy agents correlated with thromboembolism, cisplatin is expected to carry a higher risk for thromboembolic complications. Acute cerebrovascular events secondary to anti-neoplastic agents require an interdisciplinary approach, including referral to more experienced centres when needed.

Keywords: Germ cell testicular cancer, chemotherapy, cisplatin, stroke, thromboembolism
\end{abstract}

\section{Introduction}

Testicular cancer accounts for only $1 \%-2 \%$ of male cancers. However, is the most commonly diagnosed cancer in young males aged between 15 and 35 (1). Testicular germ cell tumours are considered to be one of the most curable cancers (2). The wide use of cisplatin-based chemotherapy regimens is correlated with improved outcomes. In contrast, therapyrelated morbidity remains a serious concern (3). While adverse effects such as nephrotoxicity, peripheral neuropathy, ototoxicity or pulmonary toxicity are routinely considered, vascular toxicity seems to receive less frequent attention yet remains a confronting challenge. A recent study suggests that early vascular endothelial changes in patients who underwent cisplatin-based chemotherapy may contribute to long-term cardiovascular morbidity in survivors (4). Herein, we present a case of a patient diagnosed with non-seminomatous germ cell tumour (NSGCT), who underwent adjuvant cisplatin-based chemotherapy treatment and subsequently developed an acute ischemic stroke. We present the management of this adverse effect and discuss the possible causes of this situation. We suggest that his cisplatin-based chemotherapy regimen played a role in this case.

\section{Case Presentation}

A 34-year-old male; without significant medical history or previous cardiovascular events, presented with a right-sided testicular mass. Testicular ultrasound showed a $90 \times 65 \times 55 \mathrm{~mm}$ solid mass in the right testicle. The patient's tumour markers were as follows: $\beta$-human chorionic gonadotropin ( $\beta$-hCG) of $37.42 \mathrm{mlU} / \mathrm{mL}$, $\alpha$-fetoprotein (AFP) of $17.5 \mathrm{ng} / \mathrm{mL}$ and lactic dehydrogenase (LDH) of $275 \mathrm{U} / \mathrm{L}$. Radical inguinal orchiectomy was performed, and the pathologist reported a malignant mixed germ cell tumour, composed of seminoma (90\%), yolk sac tumour (5\%) and teratoma (5\%). The pathological staging was determined as pT2. A computed tomography (CT) of the abdomen and thorax showed no signs of metastasis.

A single cycle of bleomycin-etoposide-cisplatin (BEP) chemotherapy protocol was initiated three weeks after the surgery. Tumour markers at the beginning of the chemotherapy were regressed to $1.2 \mathrm{mlU} / \mathrm{mL}$ ( $\beta$-hCG), $2.46 \mathrm{ng} / \mathrm{mL}$ (AFP) and 
$168 \mathrm{U} / \mathrm{L}(\mathrm{LDH})$. The patient received his chemotherapy and was discharged without any problem. Four days following his discharge, he developed fever, left-sided paralysis of the upper and lower extremities, and difficulty speaking. He also suffered from malaise and fatigue. He was admitted to the emergency department, and a cranial CT scan showed no signs of acute lesions. His neurological examination (NE) upon admission showed mild motor weakness in his upper (4/5) and lower $(4 / 5)$ extremities, with no sensory deficit, and an apparent loss of the left nasolabial fold. He had no aphasia, and the NE was otherwise healthy. He was admitted to the urology ward. A cranial magnetic resonance imaging (MRI) was performed, along with a biochemical examination of his blood and a complete blood count. The MRI showed sub-acute infarction areas in the right middle cerebral artery shed area (Figure 1). Later, CT angiography of the head and neck was performed, which revealed a floating thrombus in the right internal carotid artery (ICA) (Figure 2). Neither open surgical nor minimalinvasive intervention was advised. Anti-coagulant treatment with $4000 \mathrm{IU}$ of low molecular weight heparin (LMWH) every $12 \mathrm{~h}$ was ordered instead.

Several tests were done to decipher the factor underlying the arterial thrombotic event seen in this patient. An electrocardiogram at admission revealed a normal sinus rhythm. In contrast, trans-thoracic echocardiography and a 24-h Holter monitoring showed no signs of arrhythmia, patent foramen ovale or an intracardiac thrombus, excluding a cardiac cause. His $\mathrm{HbA1c}$ value was 5.8\%. A lipid profile showed no dyslipidaemia

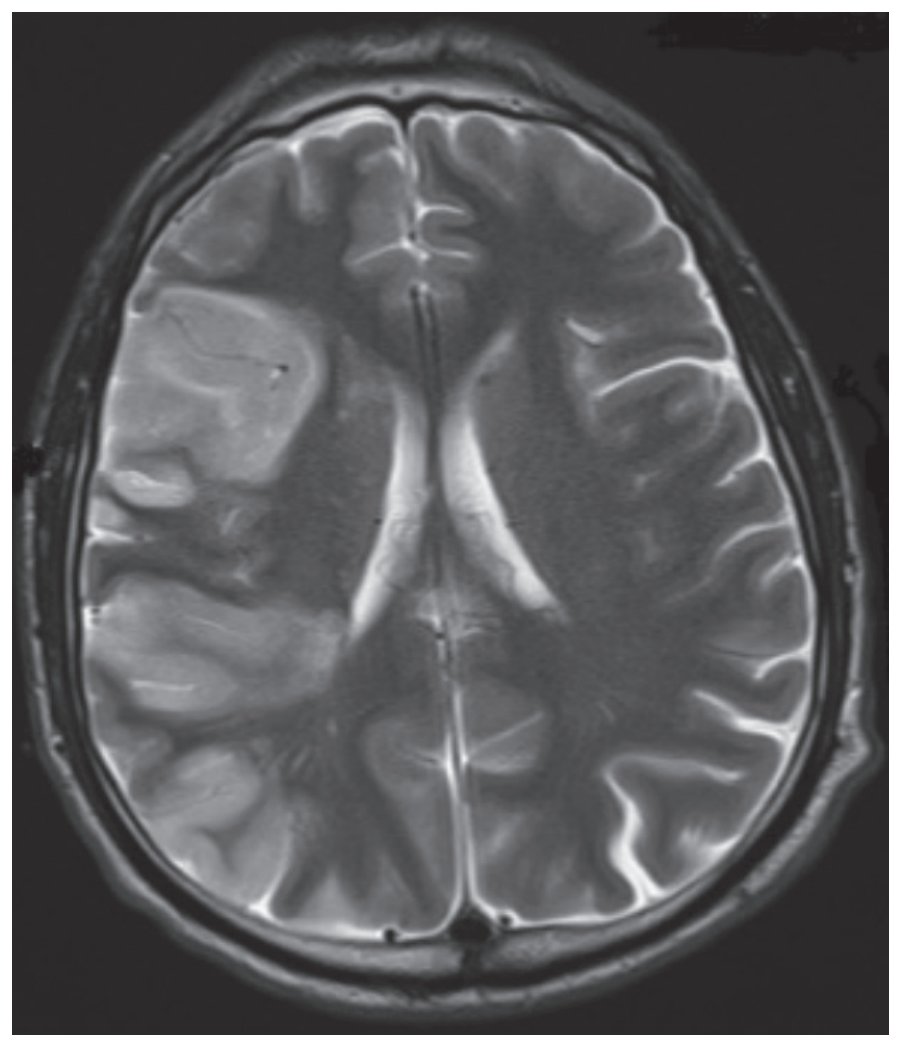

Figure 1. Magnetic resonance image of sub-acute infarction in the right middle cerebral artery shed area symptoms; low-density lipoprotein of $122 \mathrm{mg} / \mathrm{dL}$, high-density lipoprotein of $36 \mathrm{mg} / \mathrm{dL}$ and a total cholesterol level of 175 $\mathrm{mg} / \mathrm{dL}$. Anti-nuclear antibodies and lupus anti-coagulant tests, which were ordered to rule out the rheumatological disorders that could contribute to the patient's condition, were within healthy limits. $\mathrm{C} 1$ esterase inhibitor and C3 complement levels were normal, ruling out a disorder of the complement system. Gene mutations in factor V Leiden, prothrombin, C677T and A1298C polymorphisms of methylenetetrahydrofolate reductase (MTHFR), and 4G/5G polymorphism of the plasminogen activator inhibitor type 1 (PAl-1) gene were assessed by realtime polymerase chain reaction. Heterozygote mutations in prothrombin and MTHFR C677T and a homozygote 4G/4G polymorphism of PAI-1 were determined. Protein $\mathrm{C}$ and Protein $S$ deficiencies are other important causes of inherited thrombophilia cases. However, tests revealed that the activities of Protein $C$ and Protein $S$ were normal. His homocysteine level was found to be moderately elevated $(21.2 \mu \mathrm{mol} / \mathrm{L})$. His folic acid level was healthy, although his B12 level was low; therefore, he was administered intramuscular (IM) vitamin B12 injections. Von Willebrand factor antigen level was measured and was found to be elevated to $199.5 \%$. The serum magnesium level was within healthy limits.

The patient was prescribed with $4000 \mathrm{IU}$ of LMWH every $12 \mathrm{~h}, 40$ $\mathrm{mg}$ atorvastatin every $12 \mathrm{~h}$ and a vitamin B12 supplementation regimen with IM injections were initiated. Physical therapy was also advised, which he received regularly. He was encouraged to quit smoking.

One month after his initial presentation, he was seen for a follow-up examination. His NE showed only mild paraesthesia of the left foot, with no other symptoms persisting.

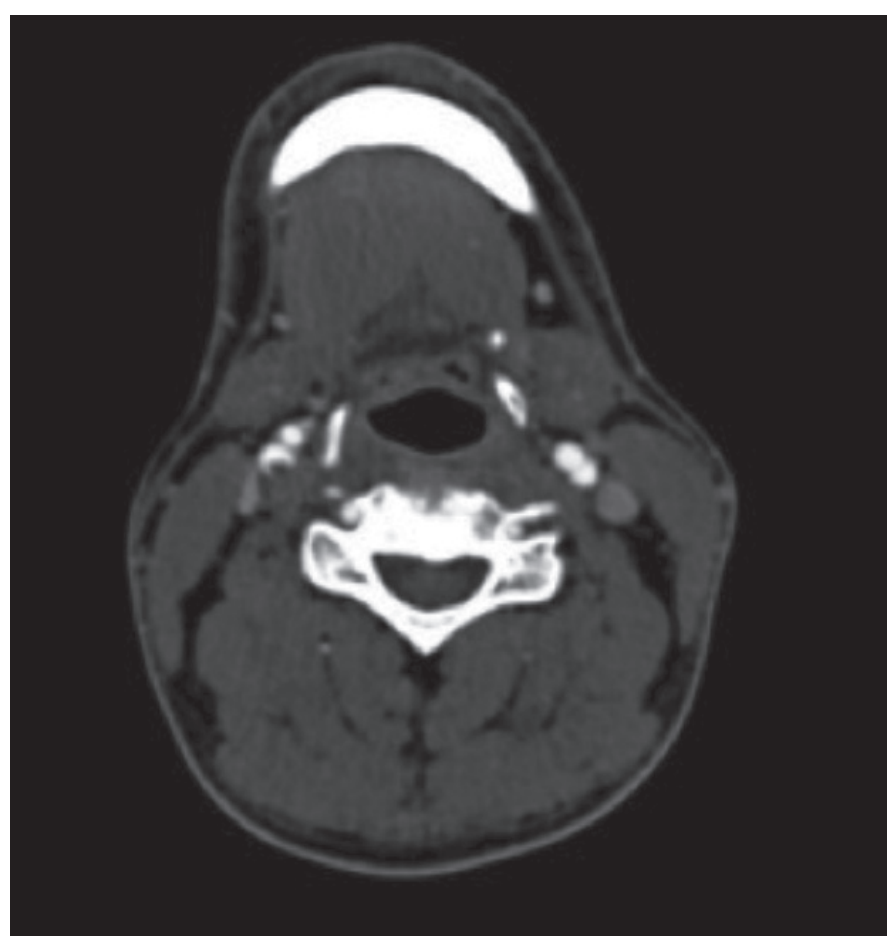

Figure 2. Computed tomography showing floating thrombus in the right internal carotid artery 


\section{Discussion}

Our patient was diagnosed with Stage IA disease, according to the tumor node metastasis classification (5). Treatment options for Stage IA NSGCT following orchiectomy include retroperitoneal lymph node dissection (RPLND), intensive follow-up with frequent CT scans, or one course of BEP for individuals who are considered at high risk of relapse (6). RPLND was not offered to the patient because of procedure-related morbidity and possible ejaculatory dysfunction. Due to socioeconomic reasons, our patient was found non-suitable for an intensive follow-up program. Hence, considering the absence of lymphovascular involvement and histologic type of the tumour, he was offered one cycle of BEP treatment.

In cancer patients, a malignancy-induced hypercoagulability state can cause thromboembolic events (7). Nonetheless, anti-cancer therapy may dramatically increase the risk of thromboembolic events by analogue mechanisms, such as the release of pro-coagulant mediators, direct endothelial injury or stimulation of tissue factor production by host cells (7). Among various chemotherapy agents that are correlated with thromboembolism, cisplatin is expected to carry a higher risk for thromboembolic complications (8). It is associated with a greater likelihood of venous thromboembolic events in daily practice. Acute arterial thromboembolic complications due to cisplatin-based chemotherapy are rare when compared with venous events (9).

Several tests and examinations were conducted to explain the pathogenesis of the ischemic stroke in our case. The patient had a history of smoking 11 cigarette packs/year. His history was negative for cerebrovascular disease and common conditions, such as diabetes mellitus or hypertension. The patient's smoking history and hyperhomocysteinemia may have made him susceptible to vascular events (10). In addition to this, the MTHFR (677) gene mutation present in our patient, can be a predisposing factor for thrombosis $(11,12)$.

Nonetheless, considering the patient's age and his rapid onset of stroke a few days after chemotherapy makes cisplatin a plausible cause. Following the vascular, metabolic and cardiac evaluation, we suggest that cisplatin-based chemotherapy was a significant etiological factor for stroke in our patient. There are case reports and a few literature reviews that indicate cisplatin-induced vascular toxicity (13). The exact pathophysiological mechanism of acute injury of cisplatin remains uncertain. Cytotoxic agents, such as cisplatin, can affect coagulation protease levels and may directly injure the endothelium (14). Owing to hypomagnesemia, cisplatin seems to be associated with serious vascular complications involving increased thrombogenicity and vascular spasm (15). Also, cisplatin has been identified as causing cardiac arrhythmias, owing to both direct drug-induced myocardial toxicity and alterations in electrolyte balance (4).

In our case, the von Willebrand factor antigen level was found to be elevated to $199.5 \%$. Previous studies also reported elevated von Willebrand factor antigen levels in patients receiving cisplatin-based chemotherapy (4). The clinical value of this finding for patients, before the administration of chemotherapy, remains unclear. High levels of von Willebrand factor antigen may have impacted on the patient's clinical condition.
Cisplatin-induced hypomagnesemia is also blamed for fatal cardiac arrhythmias (15). For this reason, the serum magnesium level of the patient was assessed but was observed to be within healthy limits.

There are multiple pathways proposed linking cisplatin therapy to vascular toxicity and thromboembolic events. It is hard to predict which patients will experience this adverse effect. Patients with known risk factors such as smoking history, older age, history of thromboembolic events and known medical conditions predisposing to thromboembolism may be under higher risk of vascular toxicity and should be monitored carefully after therapy. Prophylactic anti-thrombotic agents have been utilised in several solid cancers and showed a decrease in vascular complications (16). However, we believe that further studies are needed to justify this approach. If possible, scoring systems should be developed to risk-stratify patients and determine which patients will benefit from prophylactic anti-coagulation.

Testicular cancer cure rates are high, owing to cisplatin-based chemotherapy. Cisplatin remains an indisputable choice for oncologists and urologists worldwide. However, clinicians should be aware of less common adverse effects. When unrecognised, these side effects may result in dramatic outcomes. The decision about chemotherapy should be based on individual needs, with consideration of treatment-related risks. Acute cerebrovascular events secondary to anti-neoplastic agents require an interdisciplinary approach, including referral to more experienced centres when needed. Early cerebrovascular monitoring is of vital importance. We emphasise the correlation between the time of onset of neurological symptoms and the beginning of the thrombolytic treatment, as there is a chance of complete recovery of neurological deficits. The need and rationale for routinely screening for hypercoagulability conditions before cisplatin therapy remains unclear. Prophylactic anti-coagulant therapy should be kept in mind in high-risk patients. The pros and cons of the continuation of chemotherapy or a chemotherapy regimen change should be considered for patients who develop thrombosis.

The patient's written informed consent was obtained before the preparation of this manuscript.

\section{Acknowledgements}

Publication: The results of the study were not published in full or in part in form of abstracts.

Contribution: There is not any contributors who may not be listed as authors.

Conflict of Interest: No conflict of interest was declared by the authors.

Financial Disclosure: The authors declared that this study received no financial support.

\section{Ethics}

Informed Consent: The patient's written informed consent was obtained before the preparation of this manuscript.

Peer-review: Externally peer-reviewed. 


\section{Authorship Contributions}

Concept: B.H., M.S.Y., Design: B.H., A.A., Data Collection or Processing: B.H., M.A., A.A., Analysis or Interpretation: B.H., M.A., A.A., H.B.H., M.S.Y., Literature Search: M.A., A.A., H.B.H., Writing: B.H., M.A., H.B.H., A.A.

\section{References}

1. Curado MP, Edwards B, Shin HR, et al. Cancer incidence in five continents, volume IX. IARC Publication 2007.

2. Krege S, Beyer J, Souchon R, et al. European consensus conference on diagnosis and treatment of germ cell cancer: a report of the second meeting of the European Germ Cell Cancer Consensus group (EGCCCG): part I. Eur Urol 2008;53:478-496.

3. Hartmann JT, Kollmannsberger C, Kanz L, Bokemeyer C. Platinum organ toxicity and possible prevention in patients with testicular cancer. Int J Cancer 1999;83:866-869.

4. Nuver J, Smit AJ, van der Meer J, et al. Acute chemotherapy-induced cardiovascular changes in patients with testicular cancer. J Clin Oncol 2005;23:9130-9137.

5. Brierley JD, Gospodarowicz MK, Wittekind C. The TNM Classification of Malignant Tumours. 8th ed. 2016.

6. Albers P, Siener R, Krege S, et al. Randomized phase III trial comparing retroperitoneal lymph node dissection with one course of bleomycin and etoposide plus cisplatin chemotherapy in the adjuvant treatment of clinical stage I Nonseminomatous testicular germ cell tumors: AUO trial AH 01/94 by the German Testicular Cancer Study Group. J Clin Oncol 2008;26:2966-2972.

7. Caine GJ, Stonelake PS, Lip GY, Kehoe ST. The hypercoagulable state of malignancy: pathogenesis and current debate. Neoplasia 2002;4:465-473.
8. Khalil J, Bensaid B, Elkacemi H, et al. Venous thromboembolism in cancer patients: an underestimated major health problem. World J Surg Oncol 2015;13:204.

9. Weijl NI, Rutten MF, Zwinderman AH, et al. Thromboembolic events during chemotherapy for germ cell cancer: a cohort study and review of the literature. J Clin Oncol 2000;18:2169-2178.

10. Grysiewicz RA, Thomas K, Pandey DK. Epidemiology of ischemic and hemorrhagic stroke: incidence, prevalence, mortality, and risk factors. Neurol Clin 2008;26:871-895.

11. Cronin S, Furie KL, Kelly PJ. Dose-related association of MTHFR 677T allele with risk of ischemic stroke: evidence from a cumulative metaanalysis. Stroke 2005;36:1581-1587.

12. Supanc V, Sonicki Z, Vukasovic I, et al. The role of classic risk factors and prothrombotic factor gene mutations in ischemic stroke risk development in young and middle-aged individuals. J Stroke Cerebrovasc Dis 2014;23:e171-e176.

13. Meattini I, Scotti V, Pescini F, et al. Ischemic stroke during cisplatinbased chemotherapy for testicular germ cell tumor: case report and review of the literature. J Chemother 2010;22:134-136.

14. Lee AY. Thrombosis and cancer: the role of screening for occult cancer and recognizing the underlying biological mechanisms. Hematology Am Soc Hematol Educ Program 2006:438-443.

15. Bashir H, Crom D, Metzger $M$, et al. Cisplatin-induced hypomagnesemia and cardiac dysrhythmia. Pediatr Blood Cancer 2007;49:867-869.

16. Agnelli G, Gussoni G, Bianchini C, et al. Nadroparin for the prevention of thromboembolic events in ambulatory patients with metastatic or locally advanced solid cancer receiving chemotherapy: a randomised, placebo-controlled, double-blind study. Lancet Oncol 2009; 10:943-949. 